\title{
The Efficiency Evaluation of Information Networks in Complex Electromagnetic Environment
}

\author{
Hou Deting ${ }^{1,2}$, Wang Huabing ${ }^{1}$, Zhu Minpeng ${ }^{2+}, \mathrm{Hu}^{\mathrm{Tao}^{2}}$ \\ ${ }^{1}$ The State Key Laboratory of Complex Electromagnetic Environment \\ Effects onElectronics and Information System, Luoyang 471000, China \\ ${ }^{2}$ Information Engineering University, Zhengzhou 450001, China
}

\begin{abstract}
This paper analyzes the information network hierarchy and researches complex electromagnetic environment effects of the information network, the efficiency evaluation index system of information network in complex electromagnetic environment is established, the improved gray analytic hierarchy process is used to evaluate the performance of information network in complex electromagnetic environment. Based on the principle of indirect quantification method factor and analyzed the factors of index system characteristics, the paper proposes a reference standard for each factor, according to which the gray evaluation matrix stimulated by computer. Finally, case shows that the method can realize the quantitative information network model, which proved a reference foundation for the quantitative efficiency evaluation of information network complex electromagnetic environment.
\end{abstract}

Keywords: complex electromagnetic environment, information Network, improved grey analytical hierarchy, efficiency evaluation, indirect quantification method, index system

\section{Introduction}

With the rapid development of electronic technology and Internet technology, the information networks have become the basic element of modern society. Nevertheless, the electronic components in information networks are easily influenced in complex electromagnetic environment causing efficiency decrease. Once the efficiency is reduced, the social order will be affected. So the information network efficiency in complex electromagnetic environment is a key factor in the process of constructing information network.

Currently, there are many factors influencing information network efficiency, and some of them are difficult to quantify. The gray analytic hierarchy process (GAHP) is a useful tool to deal with such problems. It is a combined method which is the combination of grey method and analytic hierarchy process (AHP) [13]. The key of AHP is the establishment of reasonable and consistent judgment matrix, but the characters of judgment matrix are influenced by not only the experts' judgment, but also the scaling system. The consistency index of judgment matrix based on 1-9 scaling system developed by Professor Saaty[5] is big, so this paper establishes the index system of information network in complex electromagnetic environment based on the 5/5-9/1 scaling system developed by Guo Peng and Liu Shulin[6-7]. Then establishes sample value matrix according to the index efficient standard and processes data using grey theory [8-10]. The processes of advanced method are as follows.

\section{Methodology}

\subsection{Establish the comprehensive index system}

\footnotetext{
Zhu Minpeng. Tel.:13253352768.

E-mail address: $1791113619 @ \mathrm{qq} . c o m$.
} 
The efficient of information network in complex electromagnetic environment is a measurement of network services and functions based on different parameters such as: space domain, time domain, frequency domain and energy domain. Based on information network topological structure and the influence of electromagnetic environment to information network, this paper summaries node, link and survivability as three key factors of information network in complex electromagnetic environment. Then the efficiency evaluation index system of information network in complex electromagnetic environment is established showed in Fig.1.

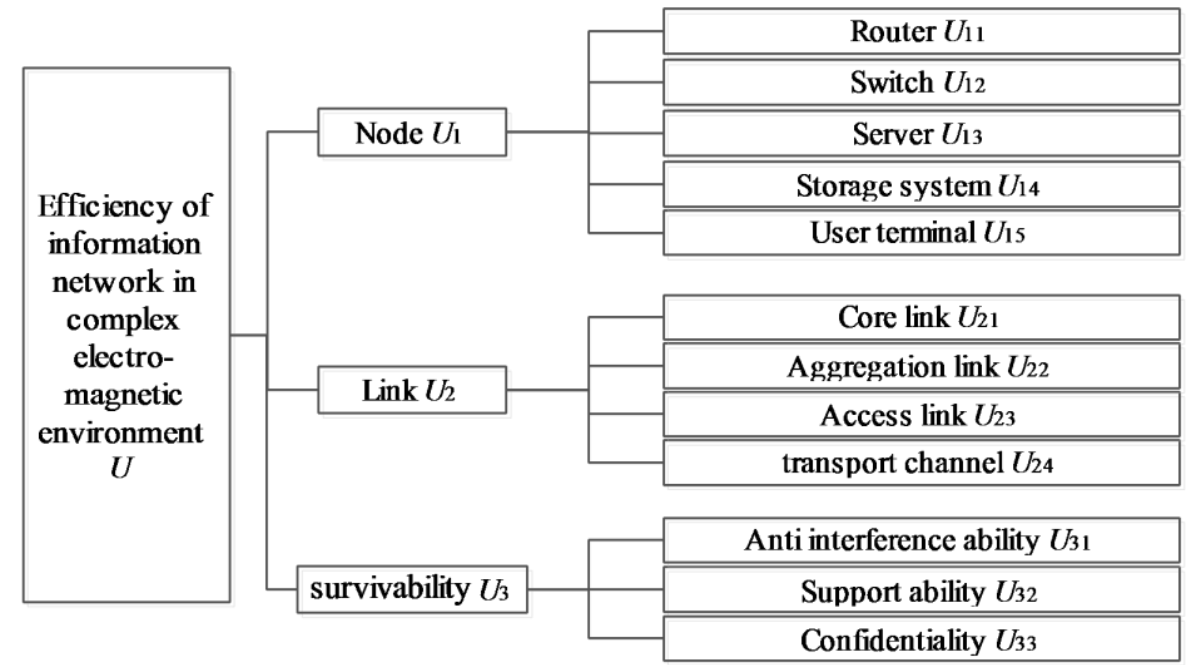

Fig.1: Efficiency evaluation index system of information network in complex electromagnetic environment

\subsection{Calculate indexes weights}

Usually the 1-9 scaling system proposed by Professor Saaty[3] is used to determine the weights of the indexes. Actually, importance is quantified by evaluating indicators, but importance difference is not as big as the number reveals. For example, according to 1-9 scaling system, the whitening values of 'moderate importance', 'strong importance' and 'demonstrated importance' are 3, 5 and 7, and that means the importance difference between two factors are 2 times, 4 times and 6 times, but in fact, people's perception of these three kinds of differences is not so large, and it can be verified by the people in the understanding of the importance of daily events in life practice. Therefore, in the establishment of judgment matrix, should adopt a more reasonable scale method and the scale value system. Here are three scaling systems in Tab.1.

Table 1: Three difference scaling system[6-7]

\begin{tabular}{|c|c|c|c|c|c|c|c|c|}
\hline Meaning & $\begin{array}{c}\text { equal } \\
\text { importance }\end{array}$ & $\begin{array}{l}i \text { th index } \\
\text { moderate } \\
\text { importance }\end{array}$ & $\begin{array}{l}\text { compared w1 } \\
\text { strong } \\
\text { importance }\end{array}$ & $\begin{array}{l}\text { ith } j \text { th index } \\
\text { demonstrated } \\
\text { importance }\end{array}$ & $\begin{array}{c}\text { extreme } \\
\text { importance }\end{array}$ & $\begin{array}{r}\text { inter } \\
\mathrm{v}\end{array}$ & $\begin{array}{l}\text { diate } \\
\text { es }\end{array}$ & $\begin{array}{c}j \text { th index } \\
\text { compared with } \\
i \text { th index }\end{array}$ \\
\hline $1-9$ & 1 & 3 & 5 & 7 & 9 & $\begin{array}{l}2 \\
6\end{array}$ & $\begin{array}{l}4 \\
8\end{array}$ & reciprocal \\
\hline $9 / 9-9 / 1$ & $9 / 9=1$ & $9 / 7=1.286$ & $9 / 5=1.8$ & $9 / 3=3$ & $9 / 1=9$ & $\begin{array}{l}9 / 8 \\
9 / 4\end{array}$ & $\begin{array}{l}9 / 6 \\
9 / 2\end{array}$ & reciprocal \\
\hline $5 / 5-9 / 1$ & $5 / 5=1$ & $6 / 4=1.5$ & $7 / 3=2.333$ & $8 / 2=4$ & $9 / 1=9$ & $\begin{array}{l}5.5 / 4 \\
7.5 / 2\end{array}$ & $\begin{array}{l}5 / 3.5 \\
5 / 1.5\end{array}$ & reciprocal \\
\hline
\end{tabular}

Through the above comparative analysis, this paper establishes the judgment matrix marked as $R_{U}$. For the element $r_{i j}$ is the importance indicator ratio of indexes $U_{i}$ and $U_{j}$, which is scored based obtained by the expert based on Tab.1.

The elements of united eigenvector corresponding to largest eigenvalue of judgment matrix are the weights of the indexes. Besides, consistency test of the relative judgment matrices is necessary, and the judgment matrix is considered to be suitable if the corresponding consistency ratio (CR) is less than $0.1^{[4]}$. The $\mathrm{CR}$ is calculated by using the formula $C R=C I / R I$, and the consistency index (CI) value is calculated by using the formula $C I=\left(\lambda_{M A X}-n\right) /(n-1)$. $\lambda_{M A X}$ is largest eigenvalue of the judgment matrix. The $R I$ (random consistency index) has given in Tab.2. 
Table 2: "5/5-9/1" random index[4]

\begin{tabular}{cccccccc}
\hline$n$ & 3 & 4 & 5 & 6 & 7 & 8 & 9 \\
\hline$R_{I}$ & 0.1690 & 0.2598 & 0.3287 & 0.3694 & 0.4007 & 0.4167 & 0.4370 \\
\hline
\end{tabular}

\subsection{Make grey evaluation}

The first step of the evaluation is establishing sample value matrix $D=\left\{d_{i j}\right\}$ which has $m \times n$ elements. The element $d_{i j}(\mathrm{i}=1,2, \cdots, n)$ is the $j$ th evaluation value of evaluation index $U_{i}$. These evaluation values can be given by the experts, experiment and simulation.

The grey level is another part in this method. Is needed to determine the number of grey number and the weight function to describe it. The evaluation grey level is usually determined on the basis of actual requirements and described by the grey number. The whitening weight functions $f_{i}(\cdot)$ corresponding to evaluation index $U_{i}$ are established according to actual requirements, and each whitening weight function should correspond to own evaluation grey level. This is the typical whitening weight function:

$$
f_{i}^{e}(x)= \begin{cases}0, & x \notin\left[x_{1}, x_{4}\right] \\ \frac{x-x_{1}}{x_{2}-x_{1}}, & x \in\left[x_{1}, x_{2}\right] \\ 1, & x \in\left[x_{2}, x_{3}\right] \\ \frac{x_{4}-x}{x_{4}-x_{3}}, & x \in\left[x_{3}, x_{4}\right]\end{cases}
$$

It can also be marked as $\left[x_{1}, x_{2}, x_{3}, x_{4}\right]$. In this paper, evaluation grey level can be the set of low, fairly low, normal, fairly high and high five degrees marked by $e(e=1,2,3,4,5)$. These whitening weight functions are $[0,9,-,-],[0,7,7,14],[0,5,5,10],[0,3,3,6],[-, 0,1,4]$.

\subsection{Calculate grey evaluation weight matrix and final result}

For the evaluation index $U_{i}$, the grey evaluation coefficient corresponding to the evaluation grey level $e$ is marked as $\delta_{i e}$, the grey evaluation coefficient corresponding to the whole evaluation grey level is marked as $\delta_{i}$, the grey evaluation weight corresponding to the whole evaluation grey level is marked as $r_{i}$. These are calculated by using the formulas as follow:

$$
\begin{gathered}
\delta_{i e}=\sum_{j=1}^{q} f_{e}\left(d_{j i}\right) \\
r_{i e}=\frac{\delta_{i e}}{\sum_{e=1}^{s} \delta_{i e}}
\end{gathered}
$$

The grey evaluation weights can form the evaluation weight vector $r_{i}=\left[r_{i 1}, r_{i 2}, \ldots, r_{i s}\right]$, then get the evaluation weight matrix $R$ and the evaluation weight vector $B$ of evaluation object:

$$
\begin{aligned}
& R=\left\lfloor\begin{array}{l}
r_{1} \\
r_{2} \\
\vdots \\
r_{q}
\end{array}\right\rfloor=\left\lfloor\begin{array}{cccc}
r_{11} & r_{12} & \cdots & r_{1 s} \\
r_{21} & r_{22} & \cdots & r_{2 s} \\
\vdots & \vdots & \ddots & \vdots \\
r_{q 1} & r_{q 2} & \cdots & r_{q s}
\end{array}\right\rfloor \\
& B=w R=\left[b_{1}, b_{2}, \cdots, b_{s}\right]
\end{aligned}
$$

This paper uses 5 levels of gray value, the first gray class is 9 , second gray class is 7 , third gray class is 5 , fourth gray class is 3 , fifth gray class is 1 , so the evaluation of grey level value vector $C=[9,7,5,3,1]$, the evaluation value of the project's general objective is $Z=B \cdot C^{T}$. According to the evaluation weight vector $B$ and evaluation value $Z$ to determine the level of evaluation object and take appropriate measures to make improvements scientifically.

\section{Example}


The information network in complex electromagnetic environment is a complex system. Selection of information network indicators will be different because of the function difference among different information networks, but the most basic aspects of the information network system evaluation generally should be contained. On the basis of establishing information network efficiency index system principles, combined with the influence of the complicated electromagnetic environment to information network, this paper uses the AHP to establish evaluation index system of the information network in the complex electromagnetic environment as shown in figure 1. The first layer is the target layer; the second layer is the factor layer including three factors: nodes, links and survivability in complex electromagnetic environment; the third layer is the index layer including twelve indexes: routers, switches, servers, storage systems, user terminals, core links, aggregation links, access links, transmission media, anti-interference ability, support capability and confidentiality.

\subsection{Establish judgment and test consistency}

According to 5/5-9/1scaling system shown in Tab. 2, establishes judgment matrix and testes its consistency. The judgment matrix of $U-U_{i}$ in ruler layer is shown in Tab.3

Table 3: Judgment matrix of $U-U_{i}$ in ruler layer

\begin{tabular}{cccccc}
\hline$U$ & $U_{1}$ & $U_{2}$ & $U_{3}$ & Weight & Lamda=3.0020 \\
\hline$U_{1}$ & 1 & 1.5 & 4 & 0.5266 & $C I=0.0009$ \\
$U_{2}$ & $2 / 3$ & 1 & 2.333 & 0.3358 & $C R=0.0059$ \\
$U_{3}$ & $1 / 4$ & $1 / 2.333$ & 1 & 0.1376 & $R I=0.1690$ \\
\hline
\end{tabular}

The $C R$ of judgment matrix is smaller than 0.1 , so the matrix is reliable and can get the weight of each factor: $w=[0.5266,0.3358,0.1376]$. Then get weight of each index in corresponding criterion: $w_{1}=[0.2318$, $0.2318,0.3873,0.0942,0.0549], w_{2}=[0.4369,0.1828,0.1304,0.2500]$ and $w_{3}=[0.4736,0.1914,0.3350]$.

\subsection{Calculate grey evaluation weight matrix}

According to information network system damage effects in complex electromagnetic environment corresponding to different thresholds, this paper quantifies core links, aggregation links, access links, transmission media, anti-interference ability, support capability and confidentiality. As for routers, switches, servers, storage systems, user terminals, can be classified by price. This paper divides effectiveness into excellent, good, medium, poor, fairly poor five degrees and get sample value matrixes by simulation using the ten point system. The sample value matrix of the three indexes in third factor is shown as follow:

$$
D_{3}=\left[\begin{array}{lllll}
6.2506 & 6.2189 & 6.9336 & 7.0542 & 7.3779 \\
5.6445 & 4.9217 & 5.4872 & 5.5262 & 4.4660 \\
3.4332 & 3.7490 & 3.5107 & 3.3373 & 3.3125
\end{array} \mid\right.
$$

According to whitening weight functions and sample value matrixes, the grey evaluation weight matrix for each index in the third factor is calculated and shown following:

$$
R_{3}=\left\lfloor\begin{array}{ccccc}
0.4381 & 0.4293 & 0.3201 & 0.1441 & 0 \\
0.3373 & 0.2853 & 0.3830 & 0.3262 & 0.2380 \\
0.2246 & 0.2853 & 0.2970 & 0.5297 & 0.7620
\end{array}\right\rfloor
$$

According to grey evaluation weight matrix and weight vector, the evaluation vector of the third factor is $U_{w 3}=w_{3} \times R_{3}=[0.3473,0.3535,0.3244,0.3081,0.3008]$. Then get $U_{w 1}=[0.2136,0.2250,0.1718,0.1264$, $0.0969] ; U_{w 2}=[0.2919,0.2831,0.2214,0.1898,0.1886]$. Gather three vectors and get the grey evaluation weight matrix $R_{U}$, then and grey evaluation vector $U_{w}$ of target $U$ :

$$
\begin{aligned}
U_{w} & =w \times R_{U}=\left[\begin{array}{l}
0.5266 \\
0.3358 \\
0.1376
\end{array}\right]^{T}\left[\begin{array}{lllll}
0.2136 & 0.2250 & 0.1718 & 0.1264 & 0.0969 \\
0.2919 & 0.2831 & 0.2214 & 0.1898 & 0.1886 \\
0.3473 & 0.3535 & 0.3244 & 0.3081 & 0.3008
\end{array}\right] \\
& =[0.2583,0.2622,0.2095,0.1727,0.1557]
\end{aligned}
$$


The overall goal $Z$ of the efficiency evaluation is $Z=U_{w} \times C^{T}=5.8810$. That means the information network efficiency in complex electromagnetic environment can be marked 5.8810 by the improved GAHP. It belongs to normal degree according to the efficiency evaluation grade.

\section{Conclusion}

This paper research the information network efficiency in complex electromagnetic environment using improved GAHP. This paper establishes the efficiency evaluation index system with hierarchical structure of information network in complex electromagnetic environment. In the construction of judgment matrix, the weight assignment is improved, then the grey system theory and analytic hierarchy process are combined to evaluate the information network in complex electromagnetic environment. As for the process of establishing the grey evaluation index matrix, most of the relevant papers don't give the reasons for index evaluated value, just scored by a few experts, so subjectivity is relatively strong. According to the basic structure of information network, this paper gives the reasons for index evaluated value to avoid the influence of human factors. Finally, the efficiency of the information network in complex electromagnetic environment is verified by an example, which provides a reference for the quantitative evaluation of the information network in the complex electromagnetic environment.

\section{Acknowledgement}

Financial support for this project is provided by the The Open Project Foundation of CEMEE State Key Laboratory under Project No.CEMEE2016K0302B.

\section{References}

[1] Qin YH, Yan WT. Research on risk assessment of underground logistics system project based on Grey Analytic Hierarchy Process[J]. International Journal of Engineering Research and Applications, 2013, 3(3):1247-1251.

[2] Deng J L. Introduction to Grey system theory[J]. Journal of Grey System, 1989, 1(1):1-24.

[3] Saaty T L. A scaling method for priorities in hierarchical structures[J]. Journal of Mathematical Psychology, 1977, 15(3):234-281.

[4] Pedrycz W, Song M. A granulation of linguistic information in AHP decision-making problems[J]. Information Fusion, 2014, 17(6):93-101.

[5] T.L Satty. Multifactor decision-making the analytic hierarchy process. University of Potts burgh, Pittburgh, PA.1988.

[6] Guo P, Zheng W. Certain Improvements in Application of AHP [J]. Systems Engineering, 1995, 13(1)28-31:.

[7] Liu S. Further Research on Index Scale Process in AHP [J]. SYSTEMS ENGINEERING ---THEORY \& PRACTICE, 1995, 1(10) 78-80.

[8] Liu S, Zhang S, Jian L, et al. Performance Evaluation of Large Commercial Aircraft Vendors [J]. Journal of Grey System, 2015, 27(1):1-11.

[9] Liu Y, Zhou B, Feng C, et al. Application of Comprehensive Evaluation Method Integrated by Delphi and GAHP in Optimal Siting of Electric Vehicle Charging Station[C]// International Conference on Control Engineering and Communication Technology. IEEE Computer Society, 2012:25-29.

[10] Gumus A T, Yayla A Y, Erkan elik, et al. A Combined Fuzzy-AHP and Fuzzy-GRA Methodology for Hydrogen Energy Storage Method Selection in Turkey[J]. Energies, 2013, 6(6):3017-3032. 